\title{
Learning Attack Strategies from Intrusion Alerts
}

\begin{abstract}
Understanding the strategies of attacks is crucial for security applications such as computer and network forensics, intrusion response, and prevention of future attacks. Though time-consuming and error-prone, manual analysis has been the dominant way to learn attack strategies from intrusion alerts. This paper presents techniques to automatically learn attack strategies from intrusion alerts. Central to these techniques is a model that represents an attack strategy as a graph of attacks with constraints on the attack attributes and the temporal order among these attacks. To learn the intrusion strategy is then to extract such a graph from a sequences of intrusion alerts. To further facilitate the analysis of attack strategies, which is essential to many security applications such as computer and network forensics and incident handling, this paper presents techniques to measure the similarity between attack strategies. The basic idea is to reduces the similarity measurement of attack strategies into error-tolerant graph isomorphism problem, and measures the similarity between attack strategies in terms of the cost to transform one strategy into another. Finally, this paper presents some experimental results, which demonstrate the potential of the aforementioned techniques.
\end{abstract}

\section{Introduction}

It has become a well-known problem that current intrusion detection systems (IDSs) produce large volumes of alerts, including both actual and false alerts. As the network performance improves and more network-based applications are being introduced, the IDSs are generating increasingly overwhelming alerts. This problem makes it extremely challenging to understand and manage the intrusion alerts, let alone respond to intrusions timely.

It is often desirable, and sometimes necessary, to understand attack strategies in security applications such as computer and network forensics and intrusion responses. For example, attack strategies may be used to profile hackers or hacking tools in computer and network forensics. As another example, it is easier to predict attacker's next move, and reduce the damage caused by intrusions, if the attack strategy is known during intrusion response. However, in practice, it usually requires that human users analyze the data collected during intrusions manually to understand the attack strategy. This process is not only time-consuming, but also error-prone. An alternative to manual analysis is to list all possible attack strategies using vulnerability analysis tools such as attack graphs $[1,36]$. However, these tools require a predefined security property so that they can use modeling checking techniques to identify possible attack sequences that may lead to the violation of the security property.

In this paper, we present techniques to automatically learn attack strategies from intrusion alerts reported by IDSs. Our approach is based on the recent advances in intrusion alert correlation [11,32]. By examining correlated intrusion alerts, our method extracts the constraints intrinsic to the attack strategy automatically. Specifically, an attack strategy is represented as a directed acyclic graph (DAG), which we call an attack strategy graph, with nodes representing attacks, edges representing the (partial) temporal order of attacks, and constraints on the nodes and edges. These constraints represent the conditions that any attack instance must satisfy in order to use the strategy. To cope with variations in attacks, we use generalization techniques to hide the differences not intrinsic to the attack strategy.

To facilitate intrusion analysis in applications such as computer and network forensics, we further develop techniques to measure the similarity between sequences of intrusion alerts based on their attack strategies. Similarity measurement of alert sequences is a fundamental problem in many security applications such as profiling 
hackers or hacking tools, identification of undetected attacks, attack prediction, and so on. To achieve this goal, we harness the results on error tolerant graph/subgraph isomorphism detection in the pattern recognition field. By analyzing the semantics and constraints in similarity measurement of alert sequences, we transform this problem into error tolerant graph/subgraph isomorphism detection.

Our contribution in this paper is three-fold. First, we develop a model to represent attack strategies as well as algorithms to extract attack strategies from correlated alerts. Second, we develop techniques to measure the similarity between sequences of alerts on the basis of the attack strategy model. Third, we perform a number of experiments to validate the proposed techniques. Our experimental results show that our techniques can successfully extract invariant attack strategies from sequences of alerts, measure the similarity between alert sequences conforming to human intuition, and identify attacks possibly missed by IDSs.

The remainder of this paper is organized as follows. The next section presents a model to represent and extract attack strategies from a sequence of correlated intrusion alerts. Section 3 discusses the methods to measure the similarity between sequences of related alerts based on their strategies. Section 4 presents the experiments we perform to validate the proposed methods. Section 5 discusses the related work, and Section 6 concludes this paper. The appendices give details of proof, analysis, and experimental results.

\section{Modeling Attack Strategies}

In this section, we present a method to represent and automatically learn attack strategies from a sequence of related intrusion alerts. Our method is developed by extending the alert correlation model by Ning, Cui, and Reeves [32], which we call the NCR model for the sake of presentation. In the following, we first give a brief overview of the NCR model, and then discuss our method.

\subsection{An Overview of the NCR Model}

The NCR model was developed to reconstruct attack scenarios from alerts reported by IDSs. It is based on the observation that "most intrusions are not isolated, but related as different stages of attacks, with the early stages preparing for the later ones" [32]. The NCR model requires the prerequisites and consequences of intrusions. The prerequisite of an intrusion is the necessary condition for the intrusion to be successful. For example, the existence of a vulnerable ftp service is the prerequisite of a ftp buffer overflow attack against this service. The consequence of an intrusion is the possible outcome of the intrusion. For example, gaining local access as root from a remote machine may be the consequence of a ftp buffer overflow attack. The NCR model then correlates two alerts if the consequence of the earlier alert prepares for the prerequisites of the later one.

The NCR model uses logical formulas, which are logical combinations of predicates, to represent the prerequisites and consequences of intrusions. For example, a scanning attack may discover UDP services vulnerable to certain buffer overflow attacks. Then the predicate UDPVulnerableToBOF (VictimIP, VictimPort) may be used to represent this discovery.

The NCR model formally represents the prerequisites and consequences of known attacks as hyper-alert types. A hyper-alert type is a triple (fact, prerequisite, consequence), where fact is a set of alert attribute names, prerequisite is a logical formula whose free variables are all in fact, and consequence is a set of logical formulas such that all the free variables in consequence are in fact. Intuitively, a hyper-alert type encodes the knowledge about the corresponding attacks. Given a hyper-alert type $T=$ (fact, prerequisite, consequence), a type $T$ hyper-alert $h$ is a finite set of tuples on fact, where each tuple is associated with an interval-based timestamp [begin_time, end_time]. The hyper-alert $h$ implies that prerequisite must evaluate to True and all the logical formulas in consequence might evaluate to True for each of the tuples.

The correlation process in the NCR model is to identify the prepare-for relations between hyper-alerts. Intuitively, it is to check if an earlier hyper-alert contributes to the prerequisite of a later one. In the formal 


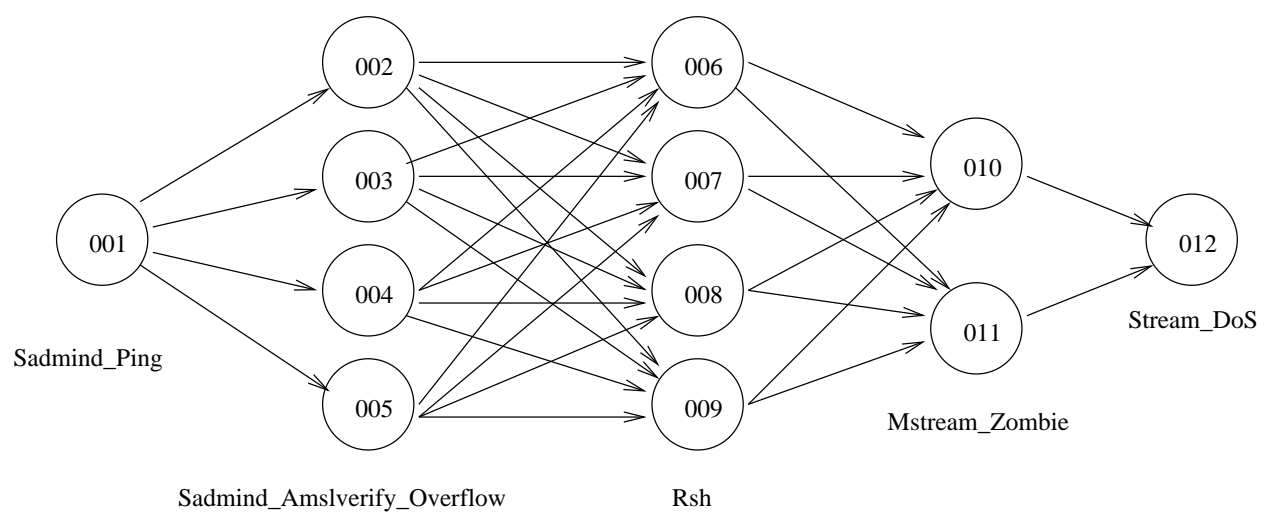

Figure 1: An example of hyper-alert correlation graph

model, this is performed through the notions of prerequisite and consequence sets. Consider a hyper-alert type $T=$ (fact, prerequisite, consequence). The prerequisite set (or consequence set) of $T$, denoted Prereq $(T)$ (or Conseq $(T)$ ), is the set of all predicates that appear in prerequisite (or consequence). Moreover, the expanded consequence set of $T$, denoted $\operatorname{ExpConseq}(T)$, is the set of all predicates that are implied by $\operatorname{Conseq}(T)$. Thus, $\operatorname{Conseq}(T) \subseteq \operatorname{ExpConseq}(T)$. Given a type $T$ hyper-alert $h$, the prerequisite set, consequence set, and expanded consequence set of $h$, denoted Prereq $(h)$, Conseq $(h)$, and ExpConseq $(h)$ ), respectively, are

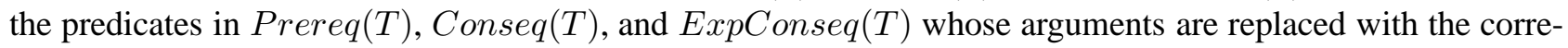
sponding attribute values of each tuple in $h$. Each element in Prereq $(h)$, Conseq $(h)$, or ExpConseq $(h)$ is associated with the timestamp of the corresponding tuple in $h$. Then hyper-alert $h_{1}$ prepares for hyper-alert $h_{2}$ if there exist $p \in \operatorname{Prereq}\left(h_{2}\right)$ and $c \in \operatorname{ExpConseq}\left(h_{1}\right)$ such that $p=c$ and c.end_time $<$ p.begin_time.

The NCR model uses a hyper-alert correlation graph to represent a set of correlated alerts. A hyper-alert correlation graph $C G=(N, E)$ is a connected directed acyclic graph (DAG), where $N$ is a set of hyper-alerts, and for each pair $n_{1}, n_{2} \in N$, there is a directed edge from $n_{1}$ to $n_{2}$ in $E$ if and only if $n_{1}$ prepares for $n_{2}$. Figure 1 shows a hyper-alert correlation graph adapted from [32]. The numbers inside the nodes represent the alert IDs, and the types of alerts are marked below the corresponding nodes.

Limitations of the NCR model. The NCR model can be used to construct attack scenarios, which are represented as hyper-alert correlation graphs, from intrusion alerts. Although such attack scenarios reflect attack strategies, they do not capture the essence of the strategies. Indeed, even with the same attack strategy, if an attacker changes certain details during attacks, the NCR model will generate very different hyper-alert correlation graphs. For example, an attacker may repeat (unnecessarily) one step in a sequence of attacks many times, and the NCR model will generate a much more complex attack scenario. As another example, if an attacker uses equivalent, but different attacks, the NCR model will generate different hyper-alert correlation graphs as well. It's then up to the user to figure out manually the common strategy behind two sequences of attacks. This fact certainly increases the overhead in intrusion alert analysis.

\subsection{Attack Strategy Graph}

In the following, we present a model to represent and automatically extract attack strategies from correlated alerts. The goal of this model is to capture the invariants in attack strategies that do not change across multiple instances of attacks.

The strategy behind a sequence of attacks is indeed about how to arrange earlier attacks to prepare for the later ones so that the attacker can reach his/her final goal. Thus, the prepare for relations between the intrusion alerts (i.e., detected attacks) is intrinsic to attack strategies. However, in the NCR model, the prepare for relations are between specific intrusion alerts; they do not directly capture the conditions that have to be met 
by related attacks. To facilitate the representation of the invariant attack strategy, we transform the prepare for relation into some common conditions that have to be satisfied by all possible instances of the same strategy. In the following, we formally represent such a conditions as an equality constraint.

Definition 1 Given a pair of hyper-alert types $\left(T_{1}, T_{2}\right)$, an equality constraint for $\left(T_{1}, T_{2}\right)$ is a conjunction of equalities in the form of $u_{1}=v_{1} \wedge \cdots \wedge u_{n}=v_{n}$, where $u_{1}, \cdots, u_{n}$ are attribute names in $T_{1}$ and $v_{1}, \cdots, v_{n}$ are attribute names in $T_{2}$, such that there exist $p\left(u_{1}, \cdots, u_{n}\right)$ and $p\left(v_{1}, \cdots, v_{n}\right)$, which are the same predicate with possibly different arguments, in $\operatorname{ExpConseq}\left(T_{1}\right)$ and $\operatorname{Prereq}\left(T_{2}\right)$, respectively. Given a type $T_{1}$ hyper-alert $h_{1}$ and a type $T_{2}$ hyper-alert $h_{2}, h_{1}$ and $h_{2}$ satisfy the equality constraint if there exist $t_{1} \in h_{1}$ and $t_{2} \in h_{2}$ such that $t_{1} \cdot u_{1}=t_{2} \cdot v_{1} \wedge \cdots \wedge t_{1} \cdot u_{n}=t_{2} \cdot v_{n}$ evaluates to True.

There may be several equality constraints for a pair of hyper-alert types. However, if a type $T_{1}$ hyper-alert $h_{1}$ prepares for a type $T_{2}$ hyper-alert $h_{2}$, then $h_{1}$ and $h_{2}$ must satisfy at least one of the equality constraints. Indeed, $h_{1}$ preparing for $h_{2}$ is equivalent to the conjunction of $h_{1}$ and $h_{2}$ satisfying at least one equivalent constraint and $h_{1}$ occurring before $h_{2}$. Assume that $h_{1}$ occurs before $h_{2}$. If $h_{1}$ and $h_{2}$ satisfy an equality constraint for $\left(T_{1}, T_{2}\right)$, then by Definition 1 , there must be a predicate $p\left(u_{1}, \cdots, u_{n}\right)$ in $\operatorname{ExpConseq}\left(T_{1}\right)$ such that the same predicate with possibly different arguments, $p\left(v_{1}, \cdots, v_{n}\right)$, is in $\operatorname{Prereq}\left(T_{2}\right)$. Since $h_{1}$ and $h_{2}$ satisfy the equality constraint, $p\left(u_{1}, \cdots, u_{n}\right)$ and $p\left(v_{1}, \cdots, v_{n}\right)$ will be instantiated to the same predicate in $\operatorname{ExpConseq}\left(h_{1}\right)$ and Prereq $\left(h_{2}\right)$. This implies that $h_{1}$ prepares for $h_{2}$. Similarly, if $h_{1}$ prepares for $h_{2}$, there must be an instantiated predicate that appears in ExpConseq $\left(h_{1}\right)$ and Prereq $\left(h_{2}\right)$. This implies that there must be a predicate with possibly different arguments in $\operatorname{Exp} \operatorname{Conseq}\left(T_{1}\right)$ and $\operatorname{Prereq}\left(T_{2}\right)$ and that this predicate leads to an equality constraint for $\left(T_{1}, T_{2}\right)$ satisfied by $h_{1}$ and $h_{2}$.

Example 1 Let us use an example from [32] to illustrate the notion of equality constraint. Consider the following hyper-alert types: SadmindPing $=(\{$ VictimIP, VictimPort $\}$, ExistsHost $($ VictimIP $),\{$ VulnerableSadmind $($ VictimIP $)\})$, and SadmindBufferOverflow $=(\{$ VictimIP, VictimPort $\}$, ExistHost $($ VictimIP $) \wedge$ VulnerableSadmind (VictimIP), $\{$ GainRootAccess (VictimIP) $\}$ ). The first hyper-alert type indicates that SadmindPing is a type of attacks that requires the existence of a host at the VictimIP to succeed, and as a result, the attacker may find out that this host has a vulnerable Sadmind service. The second hyper-alert type indicates that this type of attacks requires a vulnerable Sadmind service at the VictimIP, and as a result, the attack may gain root access. It is easy to see that there is a common predicate VulnerableSadmind in both Prereq(SadmindBufferOver flow) and ExpConseq(SadmindPing). Thus, we have an equality constraint VictimIP $=$ VictimIP for (SadmindPing, SadmindBufferOverflow), where the first VictimIP comes from SadmindPing, and the second VictimIP comes from SadmindBufferOverflow.

We observe in many occasions that one step in a sequence of attacks may trigger multiple intrusion alerts, and the number of alerts may vary in different situations. This is partially due to the existing vulnerabilities and the hacking tools. For example, unicode_shell [33], which is a hacking tool against Microsoft IIS web server, checks about 20 vulnerabilities at the scanning stage and usually triggers the same number of alerts. As another example, in the attack scenario reported in [32], the attacker tried 3 different stack pointers and 2 commands in Sadmind_Amslverify_Overflow attacks for each victim host until one attempt succeeded. Even if not necessary, an attacker may still deliberately repeat the same step multiple times to confuse IDSs and/or system administrators. However, such variations do not change the corresponding attack strategy. Indeed, these variations make the attack scenarios unnecessarily complex, and may hinder manual or automatic analysis of the attack strategy. Thus, we decide to disallow such situations in our representation of attack strategies.

In the following, an attack strategy is formally represented as an attack strategy graph.

Definition 2 Given a set $\mathcal{S}$ of hyper-alert types, an attack strategy graph over $\mathcal{S}$ is a quadruple $(N, E, T, C)$, where (1) $(N, E)$ is a connected DAG (directed acyclic graph); (2) $T$ is a mapping that maps each $n \in N$ to a hyper-alert type in $\mathcal{S}$; (3) $C$ is a mapping that maps each edge $\left(n_{1}, n_{2}\right) \in E$ to a set of equality constraints for $\left(T\left(n_{1}\right), T\left(n_{2}\right)\right)$; (4) For any $n_{1}, n_{2} \in N, T\left(n_{1}\right)=T\left(n_{2}\right)$ implies that there exists $n_{3} \in N$ such that $T\left(n_{3}\right) \neq T\left(n_{1}\right)$ and $n_{3}$ is in a path between $n_{1}$ and $n_{2}$. 


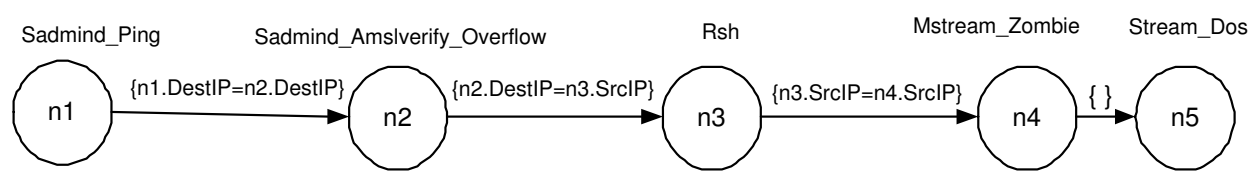

Figure 2: An example of attack strategy graph

In an attack strategy graph, each node represents a step in a sequence of related attacks. Each edge $\left(n_{1}, n_{2}\right)$ represents that a type $T\left(n_{1}\right)$ attack is needed to prepare for a successful type $T\left(n_{2}\right)$ attack. Each edge may also be associated with a set of equality constraints satisfied by the intrusion alerts. These equality constraints indicate how one attack prepares for another. Finally, as represented by condition 4 in Definition 2, the same type of attacks should be considered as one step, unless they are in different stages of the attacks.

Note that attack strategies may also be specified manually in languages such as LAMBDA [12] and STATL [15]. However, manual specification of attack strategies requires prior knowledge of the strategies, and is also time-consuming and error-prone. Tools based on modeling checking techniques (e.g., attack graphs [21, 36]) can certainly be used to build attack strategies from knowledge of individual types of attacks. However, these methods require clearly identified security properties to run the model checking tools, which may not always be available in reality. In contrast, our notion of attack strategy graph is intended to represent the strategies extracted from correlated intrusion alerts. Based on the knowledge about individual attack types, a program can automatically extract attack strategies from correlated intrusion alerts.

Now let's see an example of an attack strategy graph.

Example 2 Figure 2 is the attack strategy graph extracted from the hyper-alert correlation graph in Figure 1. The hyper-alert types are marked above the corresponding nodes, and the equality constraints are labeled near the corresponding edges. This attack strategy graph clearly shows the component attacks and the constraints that the component attacks must satisfy.

\subsubsection{Learning Attack Strategies from Correlated Intrusion Alerts}

As discussed earlier, our goal is to learn attack strategies automatically from correlated intrusion alerts. This requires that we extract the constraints intrinsic to attack strategy from alerts so that the same constraints apply to all the other instances of the same strategy.

Our strategy to achieve this goal is to process the correlated intrusion alerts in two steps. First, we aggregate intrusion alerts that belong to the same step of a sequence of attacks into one hyper-alert. For example, in Figure 1, alerts 002 through 005 are indeed attempts of the same attack with different parameters, and thus they should be aggregated as one step in the attack sequence. Second, we extract the constraints between the attack steps and represent them as an attack strategy graph. For example, after we aggregate the hyper-alerts in the first step, we may extract the attack strategy graph shown in Figure 2.

The challenge lies in the first step. Because of the variations of attacks as well as the signatures that IDSs use to recognize attacks, there is no clear way to identify intrusion alerts that belong to the same step in a sequence of attacks. In the following, we first attempt to use the attack type information to do so. The notion of aggregatable hyper-alerts is introduced formally to clarify when the same type of hyper-alerts can be aggregated.

Definition 3 Given a hyper-alert correlation graph $C G=(N, E)$, a subset $N^{\prime} \subseteq N$ is aggregatable, if (1) all nodes in $N^{\prime}$ are the same type of hyper-alerts, and (2) $\forall n_{1}, n_{2} \in N^{\prime}$, if there is a path from $n_{1}$ to $n_{2}$, then all nodes in this path must be in $N^{\prime}$.

Intuitively, in a hyper-alert correlation graph, where intrusion alerts have been correlated together, the same type of hyper-alerts can be aggregated as long as they are not used in different stages in the attack sequence. Condition 1 in Definition 3 is quite straightforward, but condition 2 deserves more explanation. Consider the same type of hyper-alerts $h_{1}$ and $h_{2}$. If $h_{1}$ prepares for a different type of hyper-alert $h^{\prime}$ (directly or indirectly), 


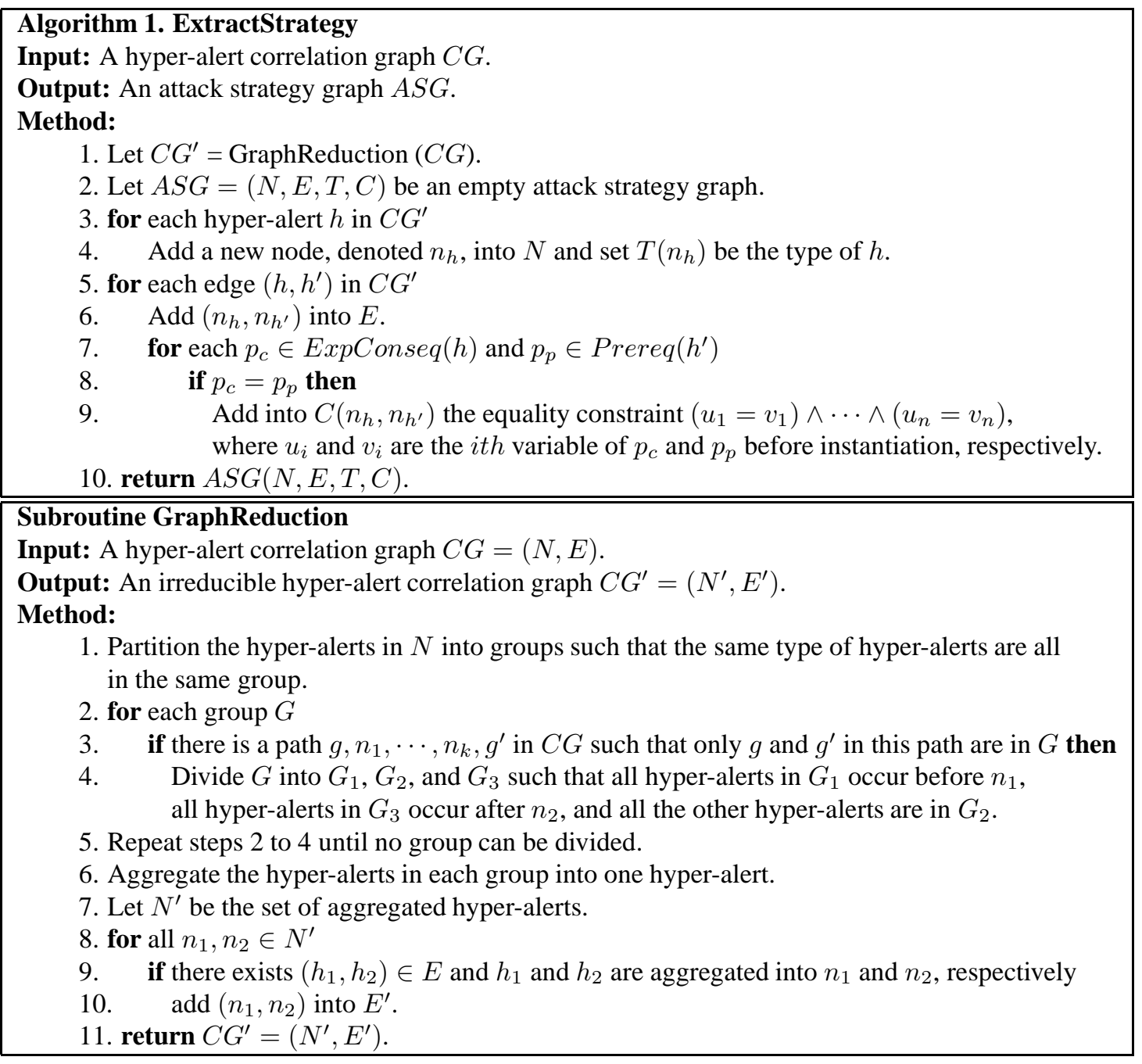

Figure 3: An algorithm to extract attack strategy graph from a hyper-alert correlation graph

and $h^{\prime}$ further prepares for $h_{2}$ (directly or indirectly), $h_{1}$ and $h_{2}$ obviously belong to different steps in the same sequence of attacks. Thus, we shouldn't allow them to be aggregated together. Although we have never observed such situations, we cannot rule out such possibilities.

Based on the notion of aggregatable hyper-alerts, the first step in learning attack strategy from a hyper-alert correlation graph is quite straightforward. We only need to identify and merge all aggregatable hyper-alerts. To proceed to the second step in strategy learning, we need a hyper-alert correlation graph in which each hyperalert represents a separate step in the attack sequence. Formally, we call such a hyper-alert correlation graph an irreducible hyper-alert correlation graph.

Definition 4 A hyper-alert correlation graph $C G=(N, E)$ is irreducible if for all $N^{\prime} \subseteq N$, where $\left|N^{\prime}\right|>1$, $N^{\prime}$ is not aggregatable.

Figure 3 shows the algorithm to extract attack strategy graphs from hyper-alert correlation graphs. The subroutine GraphReduction is used to generate an irreducible hyper-alert correlation graph, and the rest of the algorithm extracts the components of the output attack strategy graph. The steps in this algorithm are selfexplanatory; we do not repeat them in the text. Lemma 1 ensures that the output of algorithm 1 indeed satisfies the constraints of an attack strategy graph. The proof of Lemma 1 can be found in Appendix A.

Lemma 1 The output of Algorithm 1 is an attack strategy graph. 


\subsection{Dealing with Variations of Attacks}

Algorithm 1 in Figure 3 has ignored equivalent but different attacks in sequences of attacks. For example, an attacker may use either pmap_dump or Sadmind_Ping to find a vulnerable Sadmind service. As another example, an attacker may use either SadmindBufferOverflow or TooltalkBufferOverflow attack gain remote access to a host. Obviously, at the same stage of two sequences of attacks, if an attacker uses equivalent but different attacks, Algorithm 1 will return two different attack strategy graphs, though the strategies behind them are the same.

We propose to generalize hyper-alert types so that the syntactic difference between equivalent hyper-alert types is hidden. For example, we may generalize both SadmindBufferOverflow and TooltalkBufferOverflow attacks into RPCBufferOverflow.

A generalized hyper-alert type is created to hide the unnecessary difference between specific hyper-alert types. Thus, an occurrence of any of the specific hyper-alerts should imply an occurrence of the generalized one. This is to say that satisfaction of the prerequisite of a specific hyper-alert implies the satisfaction of the prerequisite of the generalized hyper-alert. Moreover, to cover all possible impact of all the specific hyper-alerts, the consequences of all the specific hyper-alert types should be included in the consequence of the generalized hyper-alert type. It is easy to see that this generalization may cause loss of information. Thus, generalization of hyper-alert types must be carefully handled so that information essential to attack strategy is not lost.

In the following, we formally clarify the relationship between specific and generalized hyper-alert types.

Definition 5 Given two hyper-alert types $T_{g}$ and $T_{s}$, where $T_{g}=\left(\right.$ fact $_{g}$, prere $_{g}$, conse $\left._{g}\right)$ and $T_{s}=\left(\right.$ fact $_{s}$, prereq $_{s}$, conseq $q_{s}$ ), we say $T_{g}$ is more general than $T_{s}$ (or, equivalently, $T_{s}$ is more specific than $T_{g}$ ) if there exists an injective mapping $f$ from fact $_{g}$ to fact $_{s}$ such that the following conditions are satisfied:

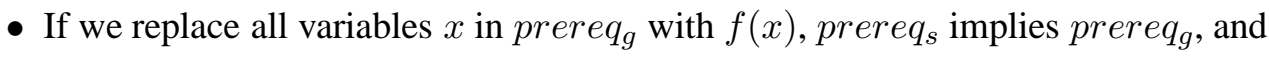

- If we replace all variables $x$ in $\operatorname{conseq}_{g}$ with $f(x)$, then all formulas in $\operatorname{conseq}_{s}$ are implied by conseq .

The mapping $f$ is called the generalization mapping from $T_{s}$ to $T_{g}$.

Example 3 Suppose the hyper-alert types SadmindBufferOverflow and TooltalkBufferOverflow are specified as follows: SadmindBufferOverflow $=(\{$ VictimIP, VictimPort $\}$, ExistHost $($ VictimIP $) \wedge$ VulnerableSadmind $($ VictimIP $),\{$ GainRootAccess $($ VictimIP $)\})$, and TooltalkBufferOverflow $=(\{$ VictimIP, VictimPort $\}$, ExistHost $($ VictimIP $) \wedge$ VulnerableTooltalk (VictimIP), \{GainRootAccess (VictimIP) $\}$ ). Assume that VulnerableSadmind (VictimIP) imply VulnerableRPC (VictimIP). Intuitively, this represents that if there is a vulnerable Sadmind service at VictimIP, then there must be a vulnerable RPC service (i.e., the Sadmind service) at VictimIP. Similarly, we assume VulnerableTooltalk (VictimIP) also implies VulnerableRPC (VictimIP). Then we can generalize both SadmindBufferOverflow and TooltalkBufferOverflow into RPCBufferOverflow $=(\{$ VictimIP $\}$, ExistHost $($ VictimIP $) \wedge$ VulnerableRPC (VictimIP), \{GainRootAccess (VictimIP) $\}$ ), where the generalization mapping is $f($ VictimIP $)=$ VictimIP.

By identifying a generalization mapping, we can specify how a specific hyper-alert can be generalized into a more general hyper-alert. Following the generalization mapping, we can find out what attribute values of a specific hyper-alert should be assigned to the attributes of the generalized hyper-alert. The attack strategy learning algorithm can be easily modified: We first generalize the hyper-alerts in the input hyper-alert correlation graph into generalized hyper-alerts following the generalization mapping, and then apply Algorithm 1 to extract the attack strategy graph.

Although a hyper-alert can be generalized in different granularities, it is not an arbitrary process. In particular, if one hyper-alert prepares for another hyper-alert before generalization, the generalized hyper-alerts should maintain the same relationship. Otherwise, the dependency between different attack stages, which is intrinsic in an attack strategy, will be lost. 
The remaining challenge is how to get the "right" generalized hyper-alert types and generalization mappings. The simplest way is to manually specify them. For example, Apache2, Back, and Crashiis are all Denial of Service attacks. We may simply generalize all of them into one WebServiceDOS. However, there are often different ways to generalize. To continue the above example, Apache 2 and Back attacks are against the apache web servers, while Crashiis is against the Microsoft IIS web server. To keep more information about the attacks, we may want to generalize Apache and Back into ApacheDOS, while generalize Crashiis and possibly other DOS attacks against the IIS web server into IISDOS. Nevertheless, this doesn't affect the attack strategy graphs extracted from correlated intrusion alerts as long as the constraints on the related alerts are satisfied.

Automatic Generalization of Hyper-Alert Types It is time-consuming and error-prone to manually generalize hyper-alert types. One way to partially automate this process is to use clustering techniques to identify the hyper-alert types that should be generalized into a common one. In our experiments, we use the bottomup hierarchical clustering [20] to group hyper-alert types hierarchically on the basis of the similarity between them, which is derived from the similarity between the prerequisites and consequences of hyper-alert types. The method used to compute the similarity is described below.

To facilitate the computation of similarity between prerequisites of hyper-alert types, we convert each prerequisite into an expanded prerequisite set, which includes all the predicates that appear or are implied by the prerequisite. Similarly, we can get the expanded consequence set. Consider two sets of predicates, denoted $S_{1}$ and $S_{2}$, respectively. We adopt the Jaccard similarity coefficient [19] to compute the similarity between $S_{1}$ and $S_{2}$, denoted $\operatorname{Sim}\left(S_{1}, S_{2}\right)$. That is, $\operatorname{Sim}\left(S_{1}, S_{2}\right)=\frac{a}{a+b+c}$, where $a$ is the number of predicates in both $S_{1}$ and $S_{2}, b$ is the number of predicates only in $S_{1}$, and $c$ is the number of predicates only in $S_{2}$.

Given two hyper-alert types $T_{1}$ and $T_{2}$, the similarity between $T_{1}$ and $T_{2}$, denoted $\operatorname{Sim}\left(T_{1}, T_{2}\right)$, is then computed as $\operatorname{Sim}\left(T_{1}, T_{2}\right)=\operatorname{Sim}\left(X P_{1}, X P_{2}\right) \times w_{p}+\operatorname{Sim}\left(X C_{1}, X C_{2}\right) \times w_{c}$, where $X P_{1}$ and $X P_{2}$ are the expanded prerequisite sets of $T_{1}$ and $T_{2}, X C_{1}$ and $X C_{2}$ are the expanded consequence sets of $T_{1}$ and $T_{2}$, and $w_{p}$ and $w_{c}=1-w_{p}$ are the weights for prerequisite and consequence, respectively. (In our experiments, we use $w_{p}=w_{c}=0.5$ to give equal weight to both prerequisite and consequence of hyper-alert types.) We may then set a threshold $t$ so that two hyper-alert types are grouped into the same cluster only if their similarity measure is greater than or equal to $t$. Appendix B includes some generalization hierarchies we encountered in our experiments.

\section{Measuring the Similarity between Attack Strategies}

In this section, we present techniques to measure the similarity between attack strategy graphs based on error tolerant graph/subgraph isomorphism detection, which has been studied extensively in pattern recognition [4,24-27]. Since the attack strategy graphs are extracted from sequences of correlated alerts, the similarity between two attack strategy graphs are indeed the similarity between the original alert sequences in terms of their strategies. Such similarity measurement is a fundamental problem in intrusion analysis; it has potential applications in incident handling, computer and network forensics, and other security management areas.

We are particularly interested in two problems. First, how similar are two attack strategies? Second, how likely is one attack strategy a part of another attack strategy? These two problems can be mapped naturally to error tolerant graph isomorphism and error tolerant subgraph isomorphism problems, respectively.

To facilitate the later discussion, we give a brief overview of error tolerant graph/subgraph isomorphism. Further details can be found in the rich literature on graph/subgraph isomorphism [4,24-27].

\subsection{Error Tolerant Graph/Subgraph Isomorphism}

In graph/subgraph isomorphism, a graph is a quadruple $G=(N, E, T, C)$, where $N$ is the set of nodes, $E$ is the set of edges, $T$ is a mapping that assigns labels to the nodes, and $C$ is a mapping that assigns labels to the 
edges. Given two graphs $G_{1}=\left(N_{1}, E_{1}, T_{1}, C_{1}\right)$ and $G_{2}=\left(N_{2}, E_{2}, T_{2}, C_{2}\right)$, a bijective function $f$ is a graph isomorphism from $G_{1}$ to $G_{2}$ if

- for all $n_{1} \in N_{1}, T_{1}\left(n_{1}\right)=T_{2}\left(f\left(n_{1}\right)\right)$;

- for all $e_{1}=\left(n_{1}, n_{1}^{\prime}\right) \in E_{1}$, there exists $e_{2}=\left(f\left(n_{1}\right), f\left(n_{1}^{\prime}\right)\right) \in E_{2}$ such that $C\left(e_{1}\right)=C\left(e_{2}\right)$, and for all $e_{2}=\left(n_{2}, n_{2}^{\prime}\right) \in E_{2}$, there exists $e_{1}=\left(f^{-1}\left(n_{2}\right), f^{-1}\left(n_{2}^{\prime}\right)\right) \in E_{1}$ such that $C\left(e_{2}\right)=C\left(e_{1}\right)$.

Given a graph $G=(N, E, T, C)$, a subgraph of $G$ is a graph $G_{s}=\left(N_{s}, E_{s}, T_{s}, C_{s}\right)$ such that (1) $N_{s} \subseteq N$, (2) $E_{s}=E \cap\left(N_{s} \times N_{s}\right)$, (3) for all $n_{s} \in N_{s}, T_{s}\left(n_{s}\right)=T\left(n_{s}\right)$, and (4) for all $e_{s} \in E_{s}, C_{s}\left(e_{s}\right)=C\left(e_{s}\right)$. Given two graphs $G_{1}=\left(N_{1}, E_{1}, T_{1}, C_{1}\right)$ and $G_{2}=\left(N_{2}, E_{2}, T_{2}, C_{2}\right)$, an injective function $f$ is a subgraph isomorphism from $G_{1}$ to $G_{2}$, if there exists a subgraph $G_{2 s}$ of $G_{2}$ such that $f$ is a graph isomorphism from $G_{1}$ to $G_{2 s}$.

As a further step beyond graph/subgraph isomorphism, error tolerant graph/subgraph isomorphism (which is also known as error correcting graph/subgraph isomorphism) is introduced to cope with noises or distortion in the input graphs. There are two approaches for error tolerant graph/subgraph isomorphism: graph edit distance and maximal common graph. In this paper, we focus on graph edit distance to study the application of error tolerant graph/subgraph isomorphism in intrusion detection.

The edit distance method assumes a set of edit operations (e.g., deletion, insertion and substitution of nodes and edges) as well as the costs of these operations, and defines the similarity of two graphs in terms of the least cost sequence of edit operations that transforms one graph into the other. We denote the edited graph after a sequence of edit operations $\Delta$ as $\Delta(G)$. Consider two graphs $G_{1}$ and $G_{2}$. The distance $D\left(G_{1}, G_{2}\right)$ from $G_{1}$ to $G_{2}$ w.r.t. graph isomorphism is the minimum sum of edit costs associated with a sequence of edit operations $\Delta$ on $G_{1}$ that leads to a graph isomorphism from $\Delta\left(G_{1}\right)$ to $G_{2}$. Similarly, the distance $D_{s}\left(G_{1}, G_{2}\right)$ from $G_{1}$ to $G_{2}$ w.r.t. subgraph isomorphism is the minimum sum of edit costs associated with a sequence of edit operations $\Delta$ on $G_{1}$ that leads to a subgraph isomorphism from $\Delta\left(G_{1}\right)$ to $G_{2}$. An error tolerant graph/subgraph isomorphism from $G_{1}$ to $G_{2}$ is a pair $(\Delta, f)$, where $\Delta$ is a sequence of edit operations on $G_{1}$, and $f$ is a graph/subgraph isomorphism from $\Delta\left(G_{1}\right)$ to $G_{2}$.

It is well known that subgraph isomorphism detection is an NP-complete problem [17]. Error tolerant subgraph isomorphism detection, which involves subgraph isomorphism detection, is also in NP and generally harder than exact subgraph isomorphism detection [25]. Nevertheless, error tolerant subgraph isomorphism has been widely applied in image processing and pattern recognition [4, 24-27]. In our application, all the attack strategy graphs we have encountered are small graphs with less than 10 nodes. We argue that it is very unlikely to have very large attack strategy graphs in practice. Thus, we believe error tolerant graph/subgraph isomorphism can be applied to measure the similarity between attack strategy graphs with reasonable response time. Indeed, we did not observe any noticeable delay in our experiments.

\subsection{Working with Attack Strategy Graphs}

To successfully use error tolerant graph/subgraph isomorphism detection techniques, we need to answer at least the following three questions. What are the edit operations on an attack strategy graph? What are reasonable edit costs of these edit operations? What is the right similarity measurement between attack strategy graphs?

All the edit operations on a labeled graph are applicable to attack strategy graphs. Specifically, an edit operation on an attack strategy graph $A S G=(N, E, T, C)$ is one of the following:

1. Inserting a node $n: \$ \rightarrow n$. This represents adding a stage into an attack strategy. This edit operation is only needed for error-tolerant graph isomorphism.

2. Deleting a node $n: n \rightarrow \$$. This represents removing a stage from an attack strategy. Note that this implies deleting all edges adjacent with $n$. 
3. Substituting the hyper-alert type of a node $n: T(n) \rightarrow t$, where $t$ is a hyper-alert type. This represents changing the attack at one stage of the attack strategy.

4. Inserting an edge $e=\left(n_{1}, n_{2}\right): \$ \rightarrow e$, where $n_{1}, n_{2} \in N$. This represents adding dependency (i.e., prepare for relation) between two attack stages.

5. Deleting an edge $e=\left(n_{1}, n_{2}\right): e \rightarrow \$$. This represents removing dependency (i.e., prepare for relation) between two attack stages.

6. Substituting the label of an edge $e=\left(n_{1}, n_{2}\right): C(e) \rightarrow c$, where $c$ is a set of equality constraints. This represents changing the way in which two attack stages are related to each other. (Note that $c$ is not necessarily a set of equality constraints for $\left(T\left(n_{1}\right), T\left(n_{2}\right)\right)$.)

These edit operations do not necessarily transform one attack strategy graph into another attack strategy graph. Indeed, a labeled graph must satisfy some constraints to be an attack strategy graph. For example, all the equality constraints in the label associated with $\left(n_{1}, n_{2}\right)$ must be valid equality constraints for $\left(T\left(n_{1}\right), T\left(n_{2}\right)\right)$. It is easy to see that the edit operations may violate some of these constraints.

One may suggest these constraints be enforced throughout the transformation of attack strategy graphs. As an additional benefit, this can be used to reduce the search space required for graph/subgraph isomorphism. However, this approach may not find the least expensive sequence of edit operations, and may even fail to find a transformation from one attack strategy graph to (the subgraph of) another. Indeed, editing distance is one way to measure the difference between attack strategy graphs; it is not necessary to require that all the intermediate edited graphs are attack strategy graph. As long as the final edited graph is isomorphic to an attack strategy graph, it is guaranteed to be an attack strategy graph. Thus, we do not require the intermediate graphs during graph transformation be attack strategy graphs.

Assignment of edit costs to the edit operations is a critical step in error tolerant graph/subgraph isomorphism. The actual costs are highly dependent on the domain in which these techniques are applied. In our application, there are multiple reasonable ways to assign the edit costs. In the following, we attempt to give some constraints that the cost assignment must satisfy.

In an attack strategy graph, a node represents a stage in an attack strategy, while an edge represents the causal relationship between two steps in the strategy. Obviously, changing the stages in an attack strategy affects the attack strategy significantly more than modifying the causal relationships between stages. Thus, the edit costs of node related operations should be significantly more expensive than those of the edge related operations.

Inserting or deleting a node implies having one more or fewer step in the strategy, while substituting a node type implies to replace the attack in one step in the strategy. Thus, inserting or deleting a node has at least the same impact on the strategy as substituting the node type. Moreover, deleting a node and inserting a node are both manipulations of a stage; there is no reason to say one operation has more impact than the other. Therefore, they should have the same cost. Both inserting and deleting an edge changes the causal relationship between two attack stages, and they should have the same impact on the attack strategy. However, substituting the label of an edge is just to change the way in which two attack stages are related. Thus, it should have less cost than edge insertion and deletion. In summary, we can derive the following constraint in edit cost assignments.

Constraint 1 Cost $_{n \rightarrow \$}=$ Cost $_{\$ \rightarrow n} \geq$ Cost $_{T(n) \rightarrow t}>>$ Cost $_{\$ \rightarrow e}=$ Cost $_{e \rightarrow \$} \geq \operatorname{Cost}_{C(e) \rightarrow c}$.

The labels in an attack strategy graph is indeed a set of equality constraints. As a result, labels are not entirely independent of each other. This further implies that edit costs for edge label substitution should not be uniformly assigned. For example, substituting an edge label $\{A, B\}$ for $\{A, C\}$ should have less cost than substituting $\{A, B\}$ for $\{C, D\}$. This observation leads to another constraint.

Constraint 2 Assume that the edit operation $C(e) \rightarrow c$ replaces $C(e)=c_{\text {old }}$ with $c_{n e w}$. The edit cost $\operatorname{Cost}_{C(e) \rightarrow c}$ should be smaller when $c_{\text {old }}$ and $c_{n e w}$ have more equality constraints in common. 
Here we give a simple way to accommodate Constraint 2. We assume there is a maximum edit cost for label substitution operation, denoted as $\operatorname{MaxCost}_{C(e) \rightarrow c}$. The edit cost of a label substitution is then $\operatorname{Cost}_{C(e) \rightarrow c}=$ $\operatorname{MaxCost}_{C(e) \rightarrow c} \times \frac{\left|c_{\text {old }} \cap c_{\text {new }}\right|}{\left|c_{\text {old }} \cup c_{\text {new }}\right|}$, where $c_{\text {old }}$ and $c_{\text {new }}$ are the labels (i.e., sets of equality constraints) before and after the operation.

Error tolerant graph/subgraph isomorphism detection techniques can conveniently give a distance between two labeled graphs, which is measured in terms of edit cost. As we discussed earlier, we use these techniques to help answer two questions: (1) How similar are two sequences of attacks in terms of their attack strategy? (2) How likely does one sequence of attacks use a part of attack strategy in another sequence of attacks? In the following, we transform the edit distance measures into more direct similarity measures.

Consider an attack strategy graph $A S G$. We refer to the distance from $A S G$ to an empty graph as the reductive weight of $A S G$, denoted as $W_{r}(A S G)$. Similarly, we refer to the distance from an empty graph to $A S G$ as the constructive weight of $A S G$, denoted $W_{c}(A S G)$.

Definition 6 Consider two attack strategy graphs $A S G_{1}$ and $A S G_{2}$. The similarity between $A S G_{1}$ and $A S G_{2}$ w.r.t. (attack) strategy is $\operatorname{Sim}\left(A S G_{1}, A S G_{2}\right)=\frac{\operatorname{Sim}\left(A S G_{1} \rightarrow A S G_{2}\right)+\operatorname{Sim}\left(A S G_{2} \rightarrow A S G_{1}\right)}{2}$, where $\operatorname{Sim}\left(A S G_{x} \rightarrow\right.$ $\left.A S G_{y}\right)=1-\frac{D\left(A S G_{x}, A S G_{y}\right)}{W_{r}\left(A S G_{x}\right)+W_{c}\left(A S G_{y}\right)}$.

Definition 7 Consider two attack strategy graphs $A S G_{1}$ and $A S G_{2}$. The similarity between $A S G_{1}$ and $A S G_{2}$ w.r.t. (attack) sub-strategy is $\operatorname{Sim}_{S u b}\left(A S G_{1}, A S G_{2}\right)=1-\frac{D_{s}\left(A S G_{1}, A S G_{2}\right)}{W_{r}\left(A S G_{1}\right)+W_{c}\left(A S G_{2}\right)}$.

Appendix $\mathrm{C}$ gives a simple analysis of the impact of edit costs on the similarity measurements. In summary, when the number of edges are not substantially more than the number of nodes, and the number of edge operations are not substantially more than the number of node operations, the similarity measure is mainly determined by the number of nodes and node operations rather than the edit costs.

\section{Experiments}

We have performed a series of experiments to study the techniques proposed in this paper. In our experiments, we used the implementation of the NCR model, the NCSU Intrusion Alert Correlator [31], to correlate intrusion alerts. Following their example, we also used GraphViz [2] to visualize graphs. In addition, we used GUB [24], A Toolkit for Graph Matching, to perform error tolerant graph/subgraph isomorphism detection and compute distances between attack strategy graphs. We used Snort [35] as our IDS sensor.

Our test data sets include the 2000 DARPA intrusion detection scenario specific data sets [28]. The data sets contain two scenarios: LLDOS 1.0 and LLDOS 2.0.2. In LLDOS1.0, the sequence of attacks includes IPsweep, probes of sadmind services, breakins through sadmind exploits, installations of DDoS programs, and finally the DDoS attack. LLDOS 2.0.2 is similar to LLDOS 1.0; however, the attacks in LLDOS 2.0.2 are more stealthy than those in LLDOS 1.0. In addition to the DARPA data sets, we also performed three sequences of attacks in an isolated network. In all these three attack sequences, the attacker started with nmap [16] scans of the victim. Then, in the first sequence, the attacker sent malformed urls [8] to the victim's Internet Information Services (IIS) to get a cmd.exe shell. In the second sequence, the attacker took advantage of the flaws of IP fragment reassembly on Windows 2000 [7] to launch a DoS attack. In the third sequence, the attacker launched a buffer overflow attack against the Internet Printing Protocol accessed via IIS 5.0 [5,9]. Further details of these attack sequences are included in Appendix D. We also used the alert sets provided along with the Intrusion Alert Correlator [31]. These alerts were generated by RealSecure Network Sensor [18] on the 2000 DARPA data sets, too. We label their alert sets with RealSecure, while label ours with Snort to distinguish between them. 


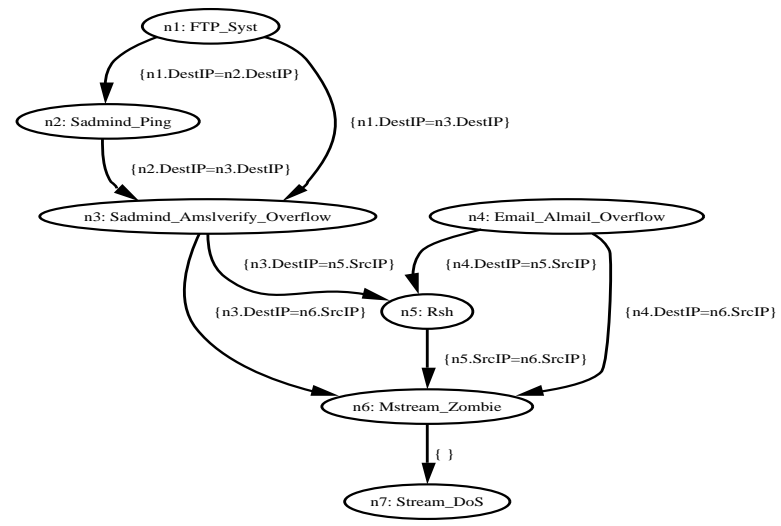

(a) LLDOS1.0 inside dataset (RealSecure)

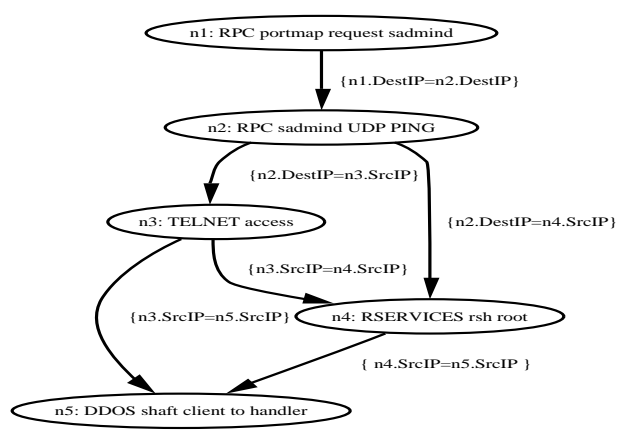

(b) LLDOS1.0 inside dataset (Snort)

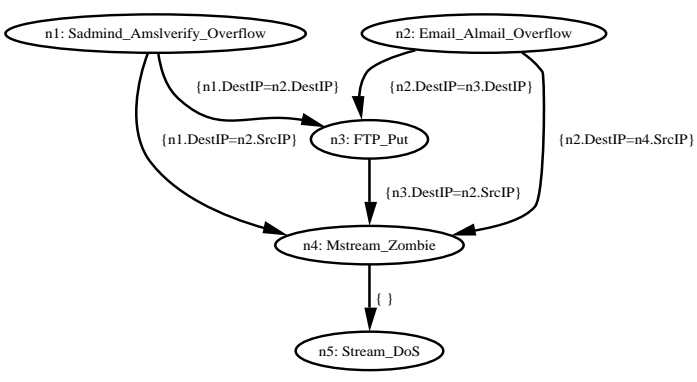

(c) LLDOS2.0.2 inside dataset (RealSecure)

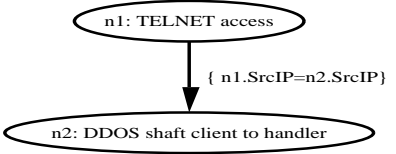

(d) LLDOS2.0.2 inside dataset (Snort)

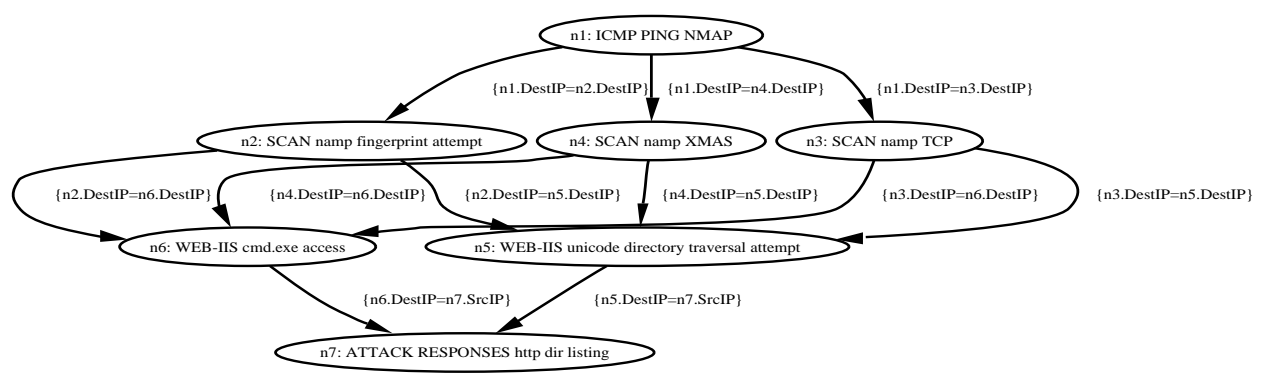

(e) WEB-IIS unicode exploits (Snort)

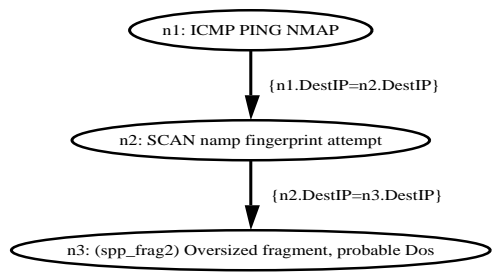

(f) jolt2 DoS attack (Snort)

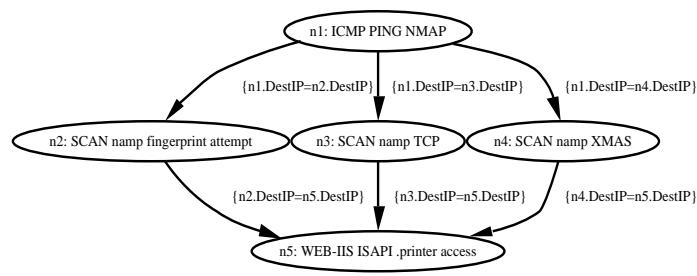

(g) WEB-IIS ISAPI .printer access (Snort)

Figure 4: Attack Strategy Graphs Extracted from Our Experiments 


\subsection{Learning Attack Strategies from Correlated Intrusion Alerts}

Our first goal is to evaluate the effectiveness of our approach on extracting the attack strategies. Figure 4 shows all of the attack strategy graphs extracted from the test data sets. The label inside each node is the node ID followed by the hyper-alert type of the node. The label of each edge describes the set of equality constraints for the hyper-alert types associated with the two end nodes.

The attack strategy graphs we extracted from LLDOS 1.0 (inside part) are shown in Figure 4(a) and 4(b). Comparing them with the description of the data set [28], we know that both Figures 4(a) and 4(b) have captured most of the attack strategy. The missing parts are due to the attacks missed by the IDSs. Since we didn't generalize variations of hyper-alert types, these graphs still have syntactic differences despite of their common strategy. (Note that the "RPC sadmind UDP PING" alert reported by Snort is indeed the "Sadmind_Amslverify_Overflow" alert by RealSecure, and the "RPC portmap sadmind request UDP" alert by Snort is the "Sadmind_Ping" alert by RealSecure.) Moreover, false alerts are also reflected in the attack strategy graphs. For example, the hyper-alert types "Email_Almail_Overflow" and "FTP_Syst" in Figure 4(a) do not belong to the attack strategy, but they are included because of the false detection.

The attack strategies extracted from LLDOS 2.0.2 are shown in Figures 4(c) and 4(d). Compared with the five phases of attack scenarios [28], it is easy to see that Figure 4(c) reveals most of the adversary's strategy. However, Figure 4(d) reveals two steps fewer than Figure 4(c). Our further investigation indicates that this is because one critical attack step, the buffer overflow attacks against sadmind service, was completely missed by Snort. Figures 4(e), 4(f), and 4(g) show the attack strategies extracted from the three sequences of attacks we performed. By comparing with the attacks, which are described in Appendix D, we can see that the stages as well as the constraints intrinsic to these attack strategies are mostly captured by these graphs.

Though showing some potential, these experimental results also reveal a limitation of the attack strategy learning method: That is, our method depends on the underlying IDSs as well as the alert correlation method. If the hyper-alert correlation graphs do not reveal the entire attack strategy, or include false alerts, the attack strategy graphs generated by our method will not be perfect. Nevertheless, our technique is intended to automate the analysis process typically performed by human analysts, who may make the same mistake if no other information is used. More research is clearly needed to mitigate the impact of imperfect IDS and correlation.

Another observation is that alerts from heterogeneous IDSs can help complete the attack strategies. For example, combining Figures 4(c) and 4(d), we know that an attacker may launch buffer overflow attacks against sadmind service and then use telnet to access the victim machine.

Note that we do not give a quantitative performance evaluation of attack strategy extraction (i.e., the false positive and false negative of the extracted attack strategies). This is because such measures are indeed determined by the underlying intrusion alert correlation algorithm. As long as correlation is performed correctly, our method can always extract the strategy reflected by the correlated alerts.

\subsection{Measuring the Similarity between Alert Sequences}

We performed some experiments to measure the similarity between the previously extracted seven attack strategy graphs. To hide the unnecessary differences between alert types, we generalized similar alert types. Due to space reasons, we do not redraw the attack strategy graphs. The generalization details are given in Appendix D. We assume the edit costs for node operations are all 10, and the edit costs for the edge operations are all 1.

Tables 1 and 2 show the similarity measurements between each pair of attack strategy graphs w.r.t. attack strategy and attack sub-strategy, respectively. Each subscript in the tables denotes the graph it represents. We notice that $\operatorname{Sim}_{S u b}\left(G_{i}, G_{j}\right)$ may not necessarily be equal to $\operatorname{Sim}_{S u b}\left(G_{j}, G_{i}\right)$.

Table 1 indicates that Figure 4(a) is more similar to Figures 4(b), and 4(c) to the other graphs. In addition, Figure 4(g) is more similar to Figures 4(e) and 4(f) than the other graphs. Based on the description of these attack sequences, we can see these similarity measures conform to human perceptions. 
Table 1: The similarity w.r.t. attack strategy between attack strategy graphs in Figure 4

\begin{tabular}{|c|c|c|c|c|c|c|c|}
\hline & $G_{4(a)}$ & $G_{4(b)}$ & $G_{4(c)}$ & $G_{4(d)}$ & $G_{4(e)}$ & $G_{4(f)}$ & $G_{4(g)}$ \\
\hline$G_{4(a)}$ & $/$ & 0.72 & 0.73 & 0.21 & 0.29 & 0.31 & 0.25 \\
\hline$G_{4(b)}$ & 0.72 & $/$ & 0.66 & 0.55 & 0.25 & 0.25 & 0.29 \\
\hline$G_{4(c)}$ & 0.73 & 0.66 & $/$ & 0.40 & 0.34 & 0.38 & 0.30 \\
\hline$G_{4(d)}$ & 0.21 & 0.55 & 0.40 & $/$ & 0.21 & 0.40 & 0.38 \\
\hline$G_{4(e)}$ & 0.29 & 0.25 & 0.34 & 0.21 & $/$ & 0.48 & 0.74 \\
\hline$G_{4(f)}$ & 0.31 & 0.25 & 0.38 & 0.40 & 0.48 & $/$ & 0.61 \\
\hline$G_{4(g)}$ & 0.25 & 0.29 & 0.30 & 0.38 & 0.74 & 0.61 & $/$ \\
\hline
\end{tabular}

Table 2: The similarity w.r.t. attack sub-strategy between attack strategy graphs in Figure 4

\begin{tabular}{|c|c|c|c|c|c|c|c|}
\hline & $G_{4(a)}$ & $G_{4(b)}$ & $G_{4(c)}$ & $G_{4(d)}$ & $G_{4(e)}$ & $G_{4(f)}$ & $G_{4(g)}$ \\
\hline$G_{4(a)}$ & $/$ & 0.72 & 0.66 & 0.31 & 0.53 & 0.31 & 0.43 \\
\hline$G_{4(b)}$ & 0.89 & $/$ & 0.67 & 0.55 & 0.61 & 0.38 & 0.51 \\
\hline$G_{4(c)}$ & 0.90 & 0.68 & $/$ & 0.40 & 0.61 & 0.38 & 0.52 \\
\hline$G_{4(d)}$ & 0.89 & 1.00 & 0.86 & $/$ & 0.79 & 0.60 & 0.73 \\
\hline$G_{4(e)}$ & 0.51 & 0.58 & 0.58 & 0.21 & $/$ & 0.48 & 0.26 \\
\hline$G_{4(f)}$ & 0.72 & 0.65 & 0.65 & 0.40 & 0.91 & $/$ & 0.89 \\
\hline$G_{4(g)}$ & 0.59 & 0.51 & 0.48 & 0.27 & 0.93 & 0.61 & $/$ \\
\hline
\end{tabular}

Table 2 shows the similarity between attack strategy graphs w.r.t. attack sub-strategy. We can see that Figures 4(b), 4(c), and 4(d) are very similar to a sub-strategy of Figure 4(a). In addition, Figure 4(d) is exactly a substrategies of Figure 4(b). Similarly, Figures 4(g) and 4(f) are both similar to sub-strategies of Figure 4(e), and Figure 4(f) is also similar to a sub-strategy of Figure 4(g). Comparing these measure values with these attack sequences, we can see these measures also conform to human perceptions.

The experiments also reveal some remaining problems that haven't been addressed by our techniques. First, the similarity measures make sense in terms of their relative values. However, we still do not understand what a specific similarity measure represents. Second, false alerts generated by IDSs have a negative impact on the measurement. It certainly requires further research to address these issues.

\subsection{Identification of Missing Detections}

Our last set of experiments is intended to study the possibility to apply the similarity measurement method to identify attacks missed by IDSs. For the sake of presentation, we first introduce two terms: precedent set and successive set. Intuitively, the precedent set of a node $n$ in an attack strategy graph is the set of nodes from which there are paths to $n$, while the successive set of $n$ is the set of nodes to which $n$ has a path. In the following, we show two examples we encountered in our experiments.

Example 4 The attack strategy graph in Figure 4(c) has no network probe phase, but Figure 4(a) does. The similarity measurement $\operatorname{Sim}_{S u b}\left(G_{4(c)}, G_{4(a)}\right)=0.90$ and $\operatorname{Sim}\left(G_{4(c)}, G_{4(a)}\right)=0.73$ indicate that these two strategies are very similar and it's very likely that Figure 4(c) is a sub-strategy of Figure 4(a). Thus, it is possible that some probe attacks are missed by the IDS when the IDS detected the attacks corresponding to Figure 4(c). Indeed, this is exactly what happened in LLDOS 2.0.2. The adversary uses some stealthy attacks (i.e., HINFO query to the DNS server) to get the information about the victim host.

Example 5 Consider Figures 4(d) and 4(b). We have $\operatorname{Sim}_{S u b}\left(G_{4(d)}, G_{4(b)}\right)=1.0$. Thus, $G_{4(d)}$ is exactly a sub-strategy of $G_{4(b)}$. By checking the LLDOS2.0.2 alerts reported by Snort, we know that there are also "RPC portmap sadmind request UDP" alerts as in Figure 4(b). However, since Snort did not detect the later buffer overflow attack, these "RPC portmap sadmind request UDP" alerts aren't correlated with the later alerts. 
We then perform the following steps, trying to identify attacks possibly missed in LLDOS 2.0.2. We pick node $n 1$ in Figure 4(d), and find its corresponding node $n 3$ in Figure 4(b), which is mapped to $n 1$ by the subgraph isomorphism. It is easy to see that in Figure 4(b), the precedent set of $n 3$ is $\{n 1 n 2\}$, and $n 1$ has the type "RPC portmap sadmind request UDP". We then go back to LLDOS 2.0.2 alerts, and find "RPC portmap sadmind request UDP" alerts before "TELNET ACCESS". By comparing the precedent set of $n 1$ in Figure 4(d) and the precedent set of $n 3$ in Figure 4(b), we suspect that "RPC sadmind UDP PING" (which corresponds to node $n 2$ in Figure 4(b)) has been missed in LLDOS 2.0.2. If we add such an alert, we may correlate it with "RPC portmap sadmind request UDP" and further with "TELNET access" in Figure 4(d). Indeed, "RPC sadmind UDP PING" is the buffer overflow attack missed by Snort in LLDOS 2.0.2.

The later part of example 5 is very similar to the abductive correlation proposed in [11]. The additional feature provided by the similarity measurement is the guidelines about what attacks may be missed. In this sense, the similarity measurement is complementary to the abductive correlation. Moreover, these examples are provided to demonstrate the potential of identifying missed attacks through measuring similarity of attack sequences. It is also possible that the attacker didn't launch those attacks. Additional research is necessary to improve the performance and reduce false identification rate.

\section{Related Work}

Our work in this paper is closely related to the recent results in intrusion alert correlation. In particular, our attack strategy model can be considered as an extension to [11] and [32]. In addition to correlating alerts together based on their relationships, we further extract the attack strategy used in the attacks, and use them to measure the similarity between sequences of alerts.

There are other alert correlation techniques. The techniques in $[10,13,37,40]$ correlate alerts on the basis of the similarities between the alert attributes. The Tivoli approach correlates alerts based on the observation that some alerts usually occur in sequence [14]. M2D2 correlates alerts by fusing information from multiple sources besides intrusion alerts, such as the characteristics of the monitored systems and the vulnerability information [29], thus having a potential to result in better results than those simply looking at intrusion alerts. The mission-impact-based approach correlates alerts raised by INFOSEC devices such as IDS and firewalls with the importance of system assets [34]. The alert clustering techniques in [22,23] use conceptual clustering and generalization hierarchy to aggregate alerts into clusters. Alert correlation may also be performed by matching attack scenarios specified by attack languages. Examples of such languages include STATL [15], LAMBDA [12], and JIGSAW [38].

Our approach to similarity measurement of attack strategies is based on error-tolerant graph/subgraph isomorphism techniques $[3,6,24,25,39]$. The early work about graph/subgraph isomorphism was presented in $[3,6,39]$. The traditional methods are $A^{*}$ based approaches [30]. The more efficient algorithms proposed recently include decomposition-based approaches [24-26] and decision tree-based approaches [24, 27].

\section{Conclusion}

In this paper, we developed techniques to extract attack strategies from correlated intrusion alerts based on the recent advances in intrusion alert correlation [11,32]. Our contributions include a model to represent and algorithms to extract attack strategies from intrusion alerts. Moreover, to accommodate variations in attacks that are not intrinsic to attack strategies, we propose to generalize different types of intrusion alerts to hide the unnecessary difference between them. Finally, we developed techniques to measure the similarity between sequences of attacks based on their strategies. Our experimental results have shown that our techniques can successfully extract invariant attack strategies from sequences of alerts, measure the similarity between alert sequences in a way conforming to human intuition, and has a potential to identify attacks missed by IDSs. 


\section{References}

[1] P. Ammann, D. Wijesekera, and S. Kaushik. Scalable, graph-based network vulnerability analysis. In Proceedings of the 9th ACM Conference on Computer and Communications Security, pages 217-224, November 2002.

[2] AT \& T Research Labs. Graphviz - open source graph layout and drawing software. http://www . research.att.com/sw/tools/graphviz/.

[3] A. T. Berztiss. A backtrack procedure for isomorphism of directed graphs. Journal of the Association for Computing Machinery, 20(3):51-64, July 1973.

[4] H. Bunke and K. Shearer. A graph distance metric based on the maximal common subgraph. Pattern Recognition Letters, 19(3-4):255-259, 1998.

[5] CERT Coordination Center. Cert advisory CA-2001-10 buffer overflow vulnerability in microsoft IIS 5.0. http://www. cert.org/advisories/CA-2001-10.html, 2001.

[6] D. G. Corneil and C. C. Gotlieb. An efficient algorithm for graph isomorphism. Journal of the Association for Computing Machinery, 17(1):51-64, January 1970.

[7] Microsoft Corporation. Microsoft security bulletin (ms00-029). http://www.microsoft.com/ technet/treeview/default.asp?url=/technet/secuority/bulletin/MS00-029. asp, 2000.

[8] Microsoft Corporation. Microsoft security bulletin (ms00-078). http://www.microsoft.com/ technet/treeview/default.asp?url=/technet/secuority/bulletin/MS00-078. asp, 2000.

[9] Microsoft Corporation. Microsoft security bulletin (ms01-023). http://www.microsoft.com/ technet/treeview/default.asp?url=/technet/secu\%rity/bulletin/MS01-023. asp, 2001.

[10] F. Cuppens. Managing alerts in a multi-intrusion detection environment. In Proceedings of the 17th Annual Computer Security Applications Conference, December 2001.

[11] F. Cuppens and A. Miege. Alert correlation in a cooperative intrusion detection framework. In Proceedings of the 2002 IEEE Symposium on Security and Privacy, May 2002.

[12] F. Cuppens and R. Ortalo. LAMBDA: A language to model a database for detection of attacks. In Proc. of Recent Advances in Intrusion Detection (RAID 2000), pages 197-216, September 2000.

[13] O. Dain and R.K. Cunningham. Building scenarios from a heterogeneous alert stream. In Proceedings of the 2001 IEEE Workshop on Information Assurance and Security, pages 231-235, June 2001.

[14] H. Debar and A. Wespi. Aggregation and correlation of intrusion-detection alerts. In Recent Advances in Intrusion Detection, LNCS 2212, pages 85 - 103, 2001.

[15] S.T. Eckmann, G. Vigna, and R.A. Kemmerer. STATL: An Attack Language for State-based Intrusion Detection. Journal of Computer Security, 10(1/2):71-104, 2002.

[16] Fyodor. Nmap free security scanner. http: / / www. insecure.org/nmap, 2003.

[17] M. R. Gary and D. S. Johnson. Computers and Intractability: A Guide to the Theory of NP-Completeness. Freeman and Company, 1979. 
[18] Internet Security Systems. RealSecure intrusion detection system. http://www. iss . net.

[19] D. A. Jackson, K. M. Somers, and H. H. Harvey. Similarity coefficients: Measures of co-occurence and association or simply measures of occurrence? The American Naturalist, 133(3):436-453, March 1989.

[20] A.K. Jain and R.C. Dubes. Algorithms for Clustering Data. Prentice Hall, 1988.

[21] S. Jha, O. Sheyner, and J.M. Wing. Two formal analyses of attack graphs. In Proceedings of the 15th Computer Security Foundation Workshop, June 2002.

[22] K. Julisch. Mining alarm clusters to improve alarm handling efficiency. In Proceedings of the 17th Annual Computer Security Applications Conference (ACSAC), pages 12-21, December 2001.

[23] Klaus Julisch and Marc Dacier. Mining intrusion detection alarms for actionable knowledge. In The 8th ACM International Conference on Knowledge Discovery and Data Mining, July 2002.

[24] B. T. Messmer. Efficient Graph Matching Algorithms for Preprocessed Model Graphs. PhD thesis, University of Bern, Switzerland, November 1995.

[25] B. T. Messmer and H. Bunke. A new algorithm for error-tolerant subgraph isomorphism detection. IEEE Transactions on Pattern Analysis and Machine Intelligence, 20(5):493-504, 1998.

[26] B. T. Messmer and H. Bunke. Efficient subgraph isomorphism detection: A decomposition approach. IEEE Transactions on Knowledge and Data Engineering, 12(2):307-323, 2000.

[27] B.T. Messmer and H. Bunke. A decision tree approach to graph and subgraph isomorphism detection. Pattern Recognition, 32(12):1979-1998, 1999.

[28] MIT Lincoln Lab. 2000 DARPA intrusion detection scenario specific datasets. http: / /www. l l . mit. edu/IST/ideval/data/2000/2000_data_index.html,2000.

[29] B. Morin, L. Mé, H. Debar, and M. Ducassé. M2D2: A formal data model for IDS alert correlation. In Proceedings of the 5th International Symposium on Recent Advances in Intrusion Detection (RAID 2002), pages 115-137, 2002.

[30] N. J. Nilsson. Principles of Artificial Intelligence. Tioga, 1980.

[31] P. Ning and Y. Cui. Intrusion alert correlator (version 0.2). http: //discovery.csc.ncsu.edu/ software/correlator/ver0.2/iac.html, 2002.

[32] P. Ning, Y. Cui, and D. S Reeves. Constructing attack scenarios through correlation of intrusion alerts. In Proceedings of the 9th ACM Conference on Computer and Communications Security, pages 245-254, Washington, D.C., November 2002.

[33] Packet storm. http: //packetstormsecurity.nl. Accessed on April 30, 2003.

[34] P.A. Porras, M.W. Fong, and A. Valdes. A mission-impact-based approach to INFOSEC alarm correlation. In Proceedings of the 5th International Symposium on Recent Advances in Intrusion Detection (RAID 2002), pages 95-114, 2002.

[35] M. Roesch. Snort - lightweight intrusion detection for networks. In Proceedings of the 1999 USENIX LISA conference, 1999.

[36] O. Sheyner, J. Haines, S. Jha, R. Lippmann, and J.M. Wing. Automated generation and analysis of attack graphs. In Proceedings of IEEE Symposium on Security and Privacy, May 2002. 
[37] S. Staniford, J.A. Hoagland, and J.M. McAlerney. Practical automated detection of stealthy portscans. Journal of Computer Security, 10(1/2):105-136, 2002.

[38] S. Templeton and K. Levitt. A requires/provides model for computer attacks. In Proceedings of New Security Paradigms Workshop, pages 31 - 38. ACM Press, September 2000.

[39] J. R. Ullmann. An algorithm for subgraph isomorphism. Journal of the Association for Computing Machinery, 23(1):31-42, 1976.

[40] A. Valdes and K. Skinner. Probabilistic alert correlation. In Proceedings of the 4th International Symposium on Recent Advances in Intrusion Detection (RAID 2001), pages 54-68, 2001.

\section{A Proof Sketch of Lemma 1}

We first prove the output of the subroutine GraphReduction is an irreducible hyper-alert correlation graph by contradiction. Consider the output $C G^{\prime}=\left(N^{\prime}, E^{\prime}\right)$ of GraphReduction. Suppose there exists $N_{s} \subseteq N^{\prime}$, where $\left|N_{s}\right|>1$, such that $N_{s}$ is aggregatable. Thus, all nodes in $N_{s}$ are the same type of hyper-alerts, and for any two different nodes $n_{1}, n_{2} \in N_{s}$, if there is a path from $n_{1}$ to $n_{2}$, then all nodes in the path are in $N_{s}$. Since $C G^{\prime}$ is aggregated from the input hyper-alert correlation graph, for all pairs of nodes $n_{1}^{\prime}$ and $n_{2}^{\prime}$, where $n_{1}^{\prime}$ and $n_{2}^{\prime}$ are aggregated into $n_{1}$ and $n_{2}$, respectively, if there exists a path from $n_{1}^{\prime}$ to $n_{2}^{\prime}$ in the input graph, all the nodes in the path must be in the group of nodes aggregated into the nodes in $N_{s}$. According to steps 3 and 4 in GraphReduction, they should have been kept in the same group and aggregated into one node in $C G^{\prime}$. This leads to a contradiction to the assumption that $n_{1}^{\prime}$ and $n_{2}^{\prime}$ are aggregated into $n_{1}$ and $n_{2}$, respectively.

Now we prove the output of Algorithm 1 is an attack strategy graph. Consider the output of Algorithm 1 $A S G=(N, E, T, C)$. It is easy to see that $T$ is a mapping that maps each $n \in N$ to a hyper-alert type, and $C$ is a mapping that maps each edge $e \in E$ to a set of equality constraints. In addition, because the input hyper-alert correlation graph is a DAG, $(N, E)$ must be a directed graph. Suppose there is a cycle $n_{1}, n_{2}, \cdots, n_{1}$ in $(N, E)$. There must exist two nodes $n_{11}, n_{12}$, and $n_{21}$ in the input hyper-alert correlation graph such that $n_{11}$ and $n_{12}$ are aggregated into $n_{1}, n_{21}$ is aggregated into $n_{2}$, and there exists a path $n_{11}, \cdots, n_{21}, \cdots, n_{12}$. However, according to the subroutine GraphReduction, $n_{11}$ and $n_{12}$ should have been put into two separate groups. Thus, $(N, E)$ cannot have any cycle. Finally, for any $n_{1}, n_{2} \in N$, since the output of GraphReduction is irreducible, if $T\left(n_{1}\right)=T\left(n_{2}\right)$, then there must exist $n_{3} \in N$ in a path between $n_{1}$ and $n_{2}$ such that $T\left(n_{3}\right) \neq T\left(n_{1}\right)$.

\section{B Automatic Generalization of Hyper-Alert Types}

This appendix shows some examples for automatic generalization of hyper-alert types. Figure 5 shows the results we obtained for the hyper-alert types in the 2000 DARPA data sets. Here the string inside the non-leaf node means Generalization Type followed by an ID. From Figure 5(b), we know that FTP_Put and Rsh can be generalized to the same type. These results were used in our later experiments when we computed the similarity measures between attack strategy graphs.

\section{Analysis of the Edit Cost Influence to Similarity Measurement}

Suppose we have two graph $G_{a}$ and $G_{b}$, which have $n_{a}$ and $n_{b}$ nodes, and $e_{a}$ and $e_{b}$ edges, respectively. Suppose we perform an error tolerant graph isomorphism from $G_{a}$ to $G_{b}$, the node operations have the same cost $C_{N}$, and edge operations have the same cost $C_{E}$, where $C_{N} \gg C_{E}$. In the sequence of edit operations, suppose there are $N_{N}$ node operations, and $N_{E}$ edge operations. Then the similarity measure can be simplified as follows: 


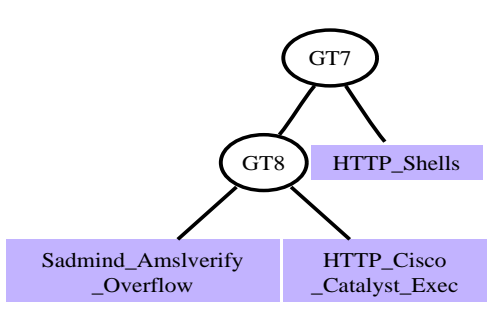

(a) Hierarchy 1

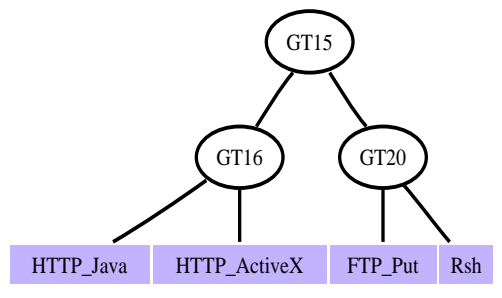

(b) Hierarchy 2

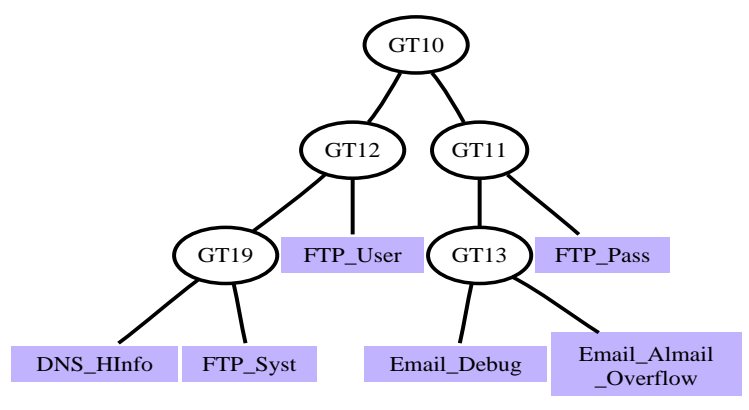

(c) Hierarchy 3

Figure 5: Generalization hierarchies for the hyper-alert types in DARPA 2000 dataset. Threshold $t=0.5$.

$$
\operatorname{Sim}\left(G_{a}, G_{b}\right)=1-\frac{D\left(G_{a}, G_{b}\right)}{W_{r}\left(G_{a}\right)+W_{c}\left(G_{b}\right)}=1-\frac{C_{N} \times N_{N}+C_{E} \times N_{E}}{C_{N} \times\left(n_{a}+n_{b}\right)+C_{E} \times\left(e_{a}+e_{b}\right)}
$$

Further let $e_{a}+e_{b}=k \times\left(n_{a}+n_{b}\right)$, and $N_{E}=s \times N_{N}$. Then we have

$$
\operatorname{Sim}\left(G_{a}, G_{b}\right)=1-\frac{C_{N} \times N_{N}+C_{E} \times s \times N_{N}}{C_{N} \times\left(n_{a}+n_{b}\right)+C_{E} \times k \times\left(n_{a}+n_{b}\right)}=1-\frac{N_{N} \times\left(C_{N}+C_{E} \times s\right)}{\left(n_{a}+n_{b}\right) \times\left(C_{N}+C_{E} \times k\right)}
$$

When $k$ and $s$ are not large, since $C_{N} \gg C_{E}$, the formula can be further simplified below:

$$
\operatorname{Sim}\left(G_{a}, G_{b}\right)=1-\frac{N_{N}}{n_{a}+n_{b}}
$$

Thus, under the above assumptions, the similarity is approximately determined by the proportion of the number of edited nodes to the total number of nodes.

\section{Further Details of The Experiments}

Attack Sequences in Our Experiments. Here we describe the three sequences of attacks performed in our experiments. Since the descriptions of the attacks in the DARPA datasets are available on-line [28], we don't repeat them in this paper.

In the first sequence of attacks (Figure 4(e)), the attacker first uses nmap [16] to scan the victim machine. (Nmap supports ping sweeps, port scans, and operating system detection.) After knowing that the OS of the 


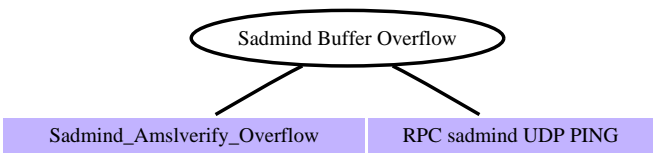

(a) Hierarchy 1

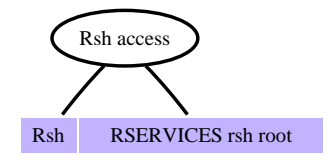

(b) Hierarchy 2

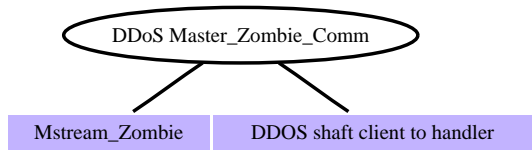

(c) Hierarchy 3

Figure 6: Additional generalizations of hyper-alert types in our experiments

victim may be windows 2000 and port 80 is open, the attacker runs a Perl script which includes 20 different malformed urls [8] against the victim machine's Internet Information Services (IIS). The output of the Perl script is a list of malformed urls to which the victim IIS is vulnerable. The attacker then selects one of the vulnerabilities to perform the actual attack and gets a cmd.exe shell on the victim machine. The attacker then uses interactive commands to list and delete some files, and finally exit the command shell. Figure 4(e) reveals this strategy.

In the second sequence of attacks (Figure 4(f)), the attacker uses nmap to scan the victim machine. However, after knowing that the OS of the victim may be windows 2000, the attacker ran a "jolt2" Perl script, which would create a DoS attack to the victim machine. (Jolt2 is a DoS attack which can take advantage of the flaws of IP fragment reassembly on Windows 2000 [7].) Figure 4(f) reveals this strategy, and because of the limitation of the Snort's signatures, it can only tell administrators "(spp_frag2) Oversized fragment, probable DoS".

In the third sequence of attacks (Figure 4(g)), the attacker uses nmap to scan the victim machine. After knowing the OS of the victim may be windows 2000 and the port 80 is open, the attacker sets up a netcat listening port on his machine, and runs a program "iis5hack" buffer overflow attack, which may result in a buffer overflow on the Internet Printing Protocol accessed via IIS 5.0 [5,9]. Following a successful iishack attack, the attaker's machine would get a reverse cmd.exe shell on the netcat listening port, and the attacker can do whatever he/she wants on the victim machine, such as listing and deleting files. In Figure 4(g), the netcat command shell attack is missing, because Snort cannot detect such attacks.

Generalization of Hyper-alert Types. The generalizations of hyper-alert types in our experiments include those in Figure 5 as well as additional ones in Figure 6. 